\title{
AN ANALYSIS OF NATURAL SCRAPIE IN! SUFFOLK SHEEP
}

\author{
A. G. DICKINSON and G. B. YOUNG \\ A.R.C. Animal Breeding Research Organisation, Edinburgh, 9 \\ J. T. STAMP and C. C. RENWICK \\ Moredun Institute, Gilmerton, Edinburgh
}

\section{INTRODUCTION}

Received 12.ii.65

NATURAL scrapie occurs in sheep of many breeds and is of world wide distribution. There have also been reports of its natural occurrence on rare occasions in goats (Chelle, r 942; Mackay and Smith, r 96I). There are good reasons for regarding the causative agent as a virus since it is filtrable (Wilson, Anderson and Smith, 1950), appears to be capable of replication (Stamp, Brotherston, Zlotnik, Mackay and Smith, 1959), can be diluted out at an appropriate level, and can be transmitted serially by inoculation in sheep (Wilson et al., 1950), goats (Gordon and Pattison, I957), hamsters (Zlotnik, I963), rats (Chandler and Fisher, I963) or mice (Chandler, I96I) or by oral transmission to sheep and goats (Pattison and Millson, $196 \mathrm{I} b$ ) or mice (Zlotnik and Rennie, 1962) or to goats by contact with sheep affected with natural scrapie (Stamp, 1962, 1965) or to mice by contact with affected mice (Dickinson, Mackay and Zlotnik, I964; Pattison, 1964).

Different strains of scrapie agent with characteristic clinical and pathological features are known in goats and mice (Pattison and Millson, I96r $a$; Zlotnik and Rennie, r963).

The agent is unusually resistant to heat and chemical treatment (Stamp et al., 1959) and the only apparent method of abolishing its activity is by autoclaving (Mackay, Smith and Stamp, 1960). The agent has not yet been positively identified by any tissue culture techniques (Mackay and Vantsis, personal communication) and hitherto no immunological characteristics of the disease have been found (Gardiner and Zlotnik, personal communication).

There is no certain method of testing for the presence or absence of the agent since techniques for isolating it have not yet been developed and since successful passage from a confirmed positive natural case is unreliable. In consequence experiments which rely for their interpretation on either an environment or host known to be free from the agent are at present impracticable and their results equivocal.

When the experiments to be described were initiated in 1955 it was widely believed by breeders that the incidence of scrapie followed a familial pattern. Our work was intended to check this belief and to test certain possibilities which might account for such a relationship. 


\section{GENERAL DESCRIPTION OF THE DISEASE AND DEFINITION OF THE PROBLEM}

Throughout this paper the term "scrapie" refers to the natural disease where no experimental challenge with a scrapie agent has occurred. When such a challenge has been made the disease will be referred to as "induced scrapie".

We define the disease in sheep as a degenerative condition of the subcortical areas of the central nervous system, the characteristic feature of which is severe intraneuronal vacuolation. Full details of the pathology have been described by Zlotnik (1958) and McDaniel and Morehouse (1963).

The clinical features of the disease can be very variable both in type and duration. Thus in Icelandic sheep (Palsson and Sigurdsson, 1958) the form of scrapie present, known locally as Rida, is primarily an incoordination of gait but without prominent pruritus. At another extreme, in hill sheep from Northern India, scrapie usually shows as severe pruritus without a protracted period of locomotor incoordination (Zlotnik and Katiyar, 196r). In Suffolk sheep in Britain, from which data in the present paper are derived unless otherwise stated, there is most often both pruritus and incoordination. However, either pruritus or incoordination can be virtually absent from the clinical syndrome in some Suffolk cases and, what is more important, extensive pathological lesions can be present in the absence of any unequivocal signs of pruritus or incoordination. More cogent still, so far as definition of the problem is concerned, because of the nonspecific nature of all except the terminal clinical signs it is possible, despite considerable experience with cases of scrapie, to make incorrect clinical diagnoses. A more complete summary of the clinical and pathological characteristics is given by Stamp (1962).

The course of the disease is extremely variable, extending from weeks to as much as years in a number of cases. One outbreak is known in a flock where the disease ran such a rapid course in a group of contemporaries that louping ill was considered during the preliminary investigation. Unless periodic clinical examinations are made of each individual in a flock, estimates of the durations of the course are of little value.

In our experience clinical scrapie is rarely present in sheep before they are two years old and, with the possible exception of the North Indian sheep (Zlotnik and Katiyar, I96I) never before 18 months old. The earliest age of sheep in which characteristic histopathological evidence of the natural disease has occurred in our data is I I months and this is exceptional.

For these reasons every sheep in these experiments which died or had to be killed over one year old has been subjected to a full neuropathological examination by Dr Zlotnik, and so too have many of the earlier deaths. One important outcome of this policy has been the 
detection of a number of incipient cases of scrapie which would otherwise have escaped notice. Roughly to per cent. of our scrapie cases were detected by this means-all showed relatively early scrapie lesions. Where pathological confirmation has been prevented by postmortem changes the animal concerned and its progeny are excluded from the data to be presented: the only exception to this is noted in the appropriate table.

It will be realised from the above that in the present state of knowledge scrapie must be defined as being fatal. An open mind must be held of the possibility of recovery until diagnostic procedures on the living animal are found. Of the several sheep suspected of recovery which have been examined none has shown the specific brain lesions when killed later, which is probably not surprising.

\section{OBJECT OF PRESENT INVESTIGATIONS}

Genetical control of a disease can be effected in many ways, the principal distinction depending on whether a pathogenic organism is involved or not. The simplest of the possibilities which was tenable at the time these experiments were formulated, though hard to reconcile in any meaningful way with the evidence on induced scrapie, was that a recessive gene was solely responsible for the disease without the involvement of a pathogen. A more plausible simple alternative, though tactically difficult to establish since exposure conditions are unknown, was that a major gene might control susceptibility of the host to a pathogenic agent. Put more generally the information required was whether any genetic relationship between cases of scrapie could be established, and if so, whether it could validly be interpreted as evidence either for the genetic origin of the disease or for genetic susceptibility to it. To achieve this aim it was essential to assess and exclude the possible involvement of pseudogenetic phenomena arising, say, from shared environments of related animals giving non-random exposure to infection, the non-genic transmission of a scrapie pathogen from mother to offspring being a special example of this.

Parry (1962) believes that his data on scrapie can be explained in terms of a recessive gene being the sole cause of the disease. The most striking evidence for this is the virtual 100 per cent. incidence in progeny from two affected parents in contrast with lower incidences in other types of mating. The present paper is largely concerned with an examination of the possibility that a recessive gene might cause scrapie.

\section{SCRAPIE IN FARM FLOCKS}

In order to understand the rationale of the present experiments it is essential to appreciate the nature and limitations of the field evidence. 
Several features such as difficulties of clinical diagnosis and widespread secrecy about the occurrence of the disease, account for certain peculiarities of the field data. If many cases have occurred in a flock it is virtually certain that at least the shepherd knows that the disease is scrapie, though the flock owner is sometimes unaware of the situation. However, a low incidence-say, 2 or 3 per cent. of breeding ewes per annum-could conceivably escape being diagnosed if the breeder has had no other experience of scrapie. Moreover, for some breeders who have not had to learn to live with it, the fear of scrapie is so great that they are inclined to dispose of animals they suspect may be developing it and consequently never know definitely one way or the other. The direct economic loss due to scrapie in commercial flocks which do not sell breeding stock is probably small. On the other hand, where the production of breeding stock for sale is the primary motive, the loss to the pedigree breeder in particular is high since the presence of scrapie in his flock, even when in low incidence, compromises the market value of all his sheep. With such a situation the prevalence of secrecy is, at least, understandable and to this must be added the difficulties of diagnosis already described, with the result that estimates of the field-incidence of the disease must be accepted with caution. We know of a flock where half of one age group developed scrapie within one year but such a high level is rare. Parry (1960) regards as high an annual observed incidence of more than to per cent. of breeding females being affected, and 4-Io per cent. as more usual in affected flocks; the same is broadly so for our field data.

As there seems to be no upper limit to the age of sheep at which scrapie can develop, exact estimates of the possible overall incidence and the incidence at each year of life are difficult to make in any group of sheep due to the commercial culling which is carried out on all farms. It has been deduced, by making allowance for the effects of commercial culling, that at least $I$ in 4 of the potential cases of scrapie are liable to occur after $4 \frac{1}{2}$ years old-the age at which about half the ewes have normally been culled (Dickinson et al., 1964c). This contrasts with only $I$ in Io being a late developing case in the data which has not been corrected for culling.

There is no reason to expect from either our own or published evidence that the males have a different actual age incidence pattern from the females though they certainly have a different culling rate.

For a number of reasons, therefore, genetic inferences which depend on detailed pedigrees, in field data subject to commercial culling, are open to serious errors due to misclassification of animals, particularly male parents, as unaffected but which may have developed scrapie had they been kept longer. Partly because of this limitation, the approach used in analysis of the data to be presented depends more on general population considerations and relatively less on the detailed accuracy of every pedigree regarding the manifestation of scrapie. 


\section{SOURCE OF FOUNDATION STOCK}

The experiments can be considered in two phases, the assembly of foundation stock and the production of progeny from experimental matings. Our approach has been to acquire two groups of Suffolk sheep: animals directly related to a case of scrapie form one group, the other comprising animals unrelated for at least a few generations to scrapie cases and from floeks where the disease had not been known to occur for many years. The two groups are referred to respectively as OS_-" off-scrapie "-and OF_-6" off-free".

The easiest and most reliable group to assemble were cases with an affected dam. Many of these came in as lambs being nursed by their affected mother-hence the problem of inaccurate flock pedigree records was virtually bypassed. This latter problem must be greatest with progeny from affected sires but this particular type constitute less than half of the OS group and for reasons which will emerge they have contributed little to the subsequent analyses. Only 6 per cent. of our OS stock are known to have both parents affected. Nineteen flocks of origin are represented in the OS group with a combined total of about 700 adult sheep during the years of close observation and approximately 7 per cent. of these individuals developed scrapie.

The search for OF stock raised certain difficulties. To have included stock from flocks with uncertain histories of supposed freedom from scrapie would be pointless. Our cronditions for inclusion had to be stringent and involved an assessment of the reliability of flock records, the trustworthiness of the owner and his ability to have recognised scrapie had it occurred: four flocks meeting these conditions have been involved. Stock from a fifth flock which was vouched for by an independent person and included because of our shortage of OF stock were initially present among the OF group: a full enquiry subsequent to the appearance of a case of scrapie brought sufficient evidence to light to necessitate the culling of this entire group. The four flocks have totalled 600 adults during our period of observation and scrapie has not appeared in any of them. However despite the careful screening, scrapie could have occurred unnoticed in these flocks, for reasons already given. Therefore as an initial proving period, OF ewes were kept loosely isolated from OS ewes for four years after purchase. The isolation simply amounted to grazing in separate fields, which for the OF stock had no recent history of being used for sheep; unavoidable contact came from the flock group which was later excluded, from mating with OS rams and indirectly via the same stockman, sheepdogs and vehicles attending both groups.

\section{INCIDENCE OF SCRAPIE IN FOUNDATION STOCK}

Fig. I shows the age incidence data for the three types OF, OS (dam affected) and OS (sire affected). Animals where both parents are known to be affected are excluded. 
The OS (sire affected) group has an average incidence of 29 per cent. among animals at least $4 \frac{1}{2}$ years old or having developed scrapie before that age. This average value cannot form the basis of any deductions since one of the sires has a much higher proportion of

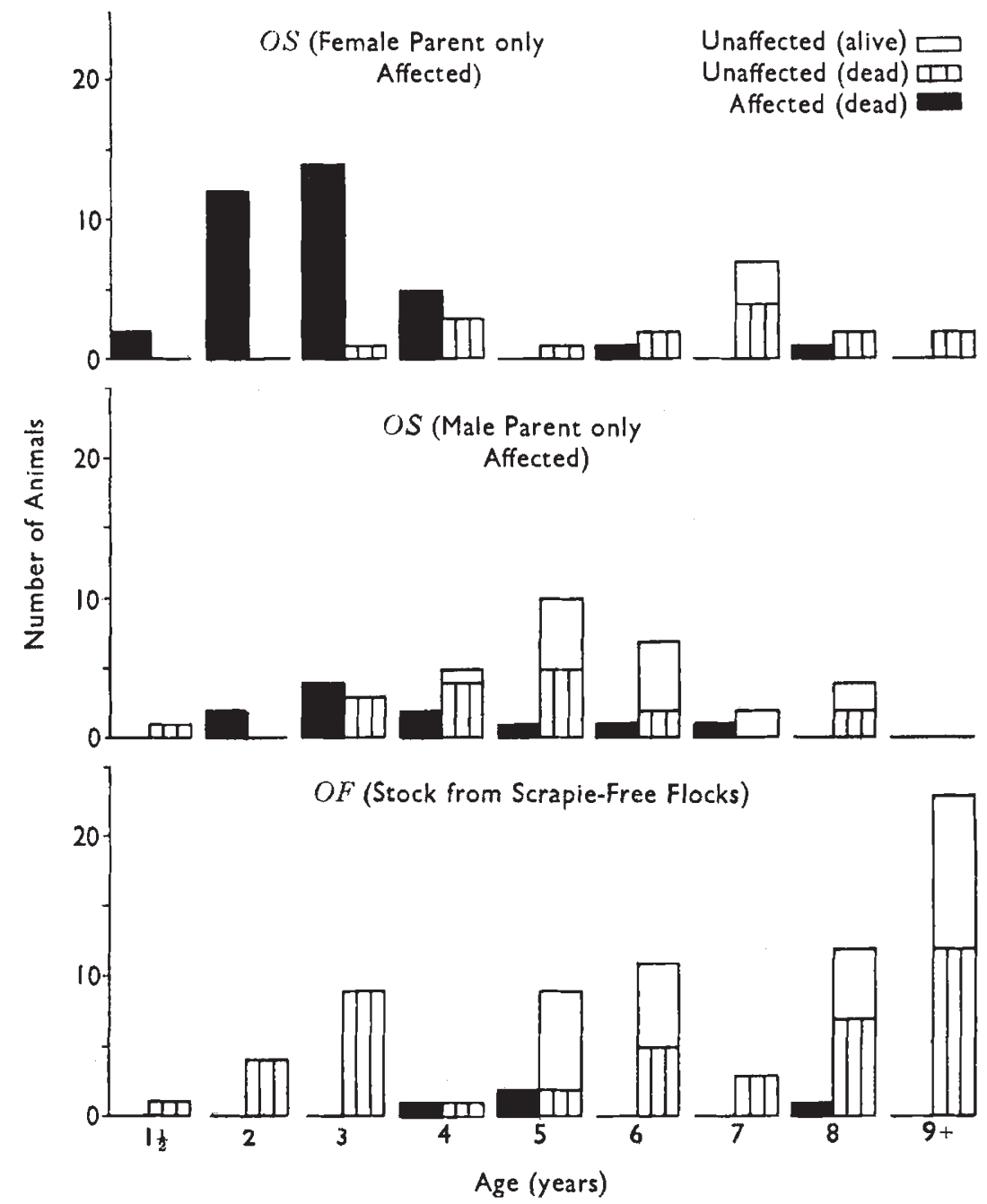

FIG. 1.-The distribution of affected and unaffected Suffolk sheep, mainly ewes, in purchased foundation stock. The classification of all the dead animals has been confirmed by neuropathological examination.

affected offspring than the others ( 70 and 12 per cent. respectively: $\mathrm{P}<0 \cdot 02)$. It is tempting to try to explain away this apparent heterogeneity in terms of unreliable pedigree records associated with some of the sires so giving us the wrong lambs but we can find no adequate evidence for this. Alternatively, it may be contended that for the deviant sire group several of the dams were actual or potential scrapie cases but unrecognised as such. However, there is some unrelated 
evidence of similar heterogeneity between progeny from a different array of sires in experimental matings, which adds to the cogency of the above result as a valid case of sire heterogeneity but no firm conclusions can be drawn.

With the OS (dam affected) group the incidence among stock born at least $4 \frac{1}{2}$ years ago has reached the high level of 66 per cent.

Among the OF group, no case of scrapie has occurred before $4 \frac{1}{2}$ years old. Of the four cases which developed later there are good grounds for excluding two. One was the only egg donor we have and was operated on contemporaneously with scrapie stock: the surgical procedures could easily have produced an experimental challenge with scrapie agent. The other was one of four OF animals placed at 2 years old in maximum lifetime contact with cases of natural scrapie. Excluding these two special cases, the OF incidence is under 3 per cent., which is very low considering that this group was not subject to any culling. This low value coupled with the unusually late development of the disease in the isolated cases, is regarded as support for the assessment of the flocks of origin as being virtually free from scrapie.

The age distribution of scrapie cases in the OF groups is quite different from that in the OS group and the difference is of the type which would be expected if contagion were involved, with the OF group not encountering the pathogen until a much later age than the one at which OS stock encountered it.

\section{SCRAPIE INCIDENCE IN PROGENY OF EWES FROM SCRAPIE-FREE FLOCKS}

The unaffected OF ewes have been mated with one or more of ten Suffolk rams some of which were known to be related to affected animals. A summary of the outcome of these matings is given in fig. 2 the purpose of which is to show the proportion of OF ewes which have left at least one affected offspring. The more progeny a ewe has, the more reliable will be the estimate of the proportion which can leave affected offspring. From fig. 2 it is concluded that at least 50 per cent. of them can do so since over half of the ewes having four or more progeny left an affected offspring. If a recessive gene were the sole cause of the disease there would be no alternative but to deduce that at least half of the OF stock were heterozygotes, most of the remainder being dominant homozygotes and a few, say 5 per cent., being homozygous recessives some of which were not observed to develop the disease. Such a mixture is hard to reconcile with population theory unless an assumption of homozygote inviability is resorted to and for which there is no independent evidence whatsoever. Moreover, assuming that mating is at random with respect to any genes affecting scrapie, this high frequency of heterozygotes in our OF sample should be matched by a scrapie incidence on the farms of origin of at least 25 per cent. and this is not so. 
The results shown in fig. 2 considered in isolation do not rule out other simple genetic explanations such as a dominant gene but for reasons which will emerge the most likely explanation is in terms of a pathogen, not encountered on the 4 farms but present in some or all of the experimental matings.

\section{INCIDENCE OF SCRAPIE IN PROGENY OF AFFECTED EWES}

A similar conclusion stems from the OS (dam affected) data (fig. I) but for quite different reasons.

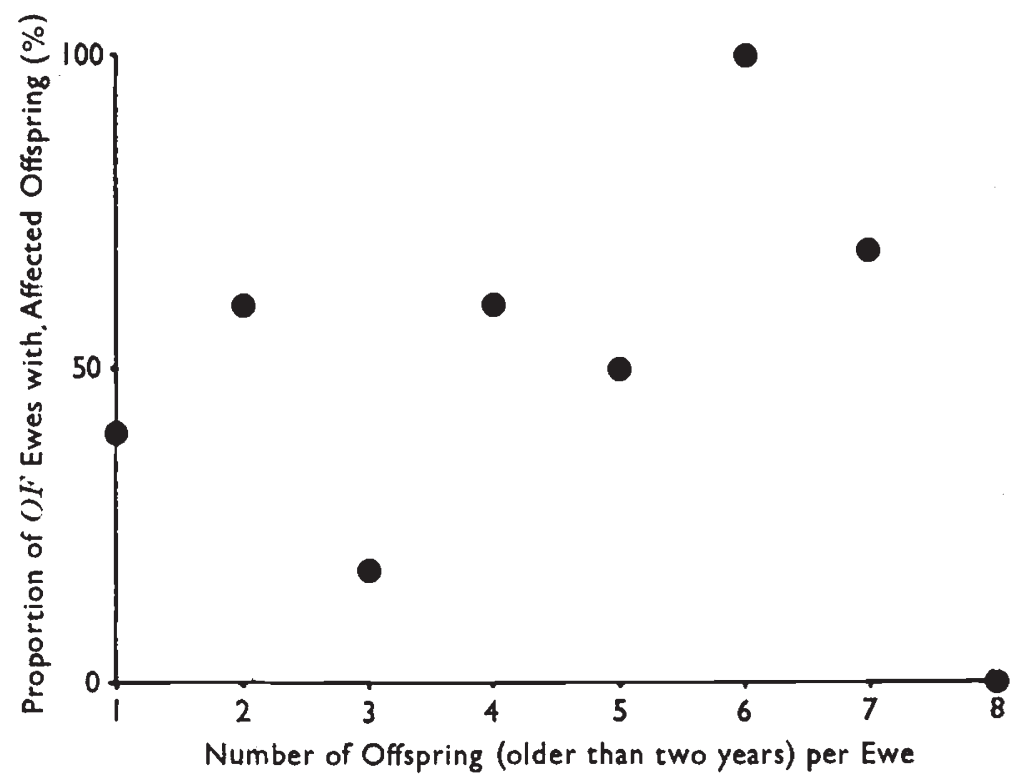

Fic. 2.-The presence of scrapie in the progeny of 37 unaffected ewes puchased from scrapie-free flocks (OF), classified according to progeny group size.

It is impossible to reconcile the high incidence of scrapie with the notion of a recessive gene unless certain qualifications are introduced since 50 per cent. is the highest Mendelian expectation possible, even after making the unlikely assumption that all unaffected rams were heterozygotes-and the observed 66 per cent. deviates even from this $(\mathrm{P}<0 \cdot 05)$. It would have to be assumed that a proportion of the male parents on the farms of origin, all thought to be unaffected, had been misclassified and were in fact potential or actual cases of scrapie and were homozygous recessives. It is quite feasible that this has happened in some cases but, in the light of the generally low incidence of scrapie and the specific exclusion of rams known to be affected, it is unlikely that misclassification occurred frequently. Also, since at least 23 ram progeny groups are represented and the average progeny group size in the OS (dam affected) class is less than 2.5 
animals, occasional misclassification of the rams can only have a very limited effect.

When these data, with allowance for misclassification, are considered in terms of known laws of population structure for a recessive gene, the outcome is shown in fig. 3. (The term " misclassification rate" requires some explanation: it denotes the proportion of supposed homozygous recessive rams in any particular population which have been wrongly classified as unaffected: the absolute number of animals misclassified for a given sample size varies with the allele frequency.)

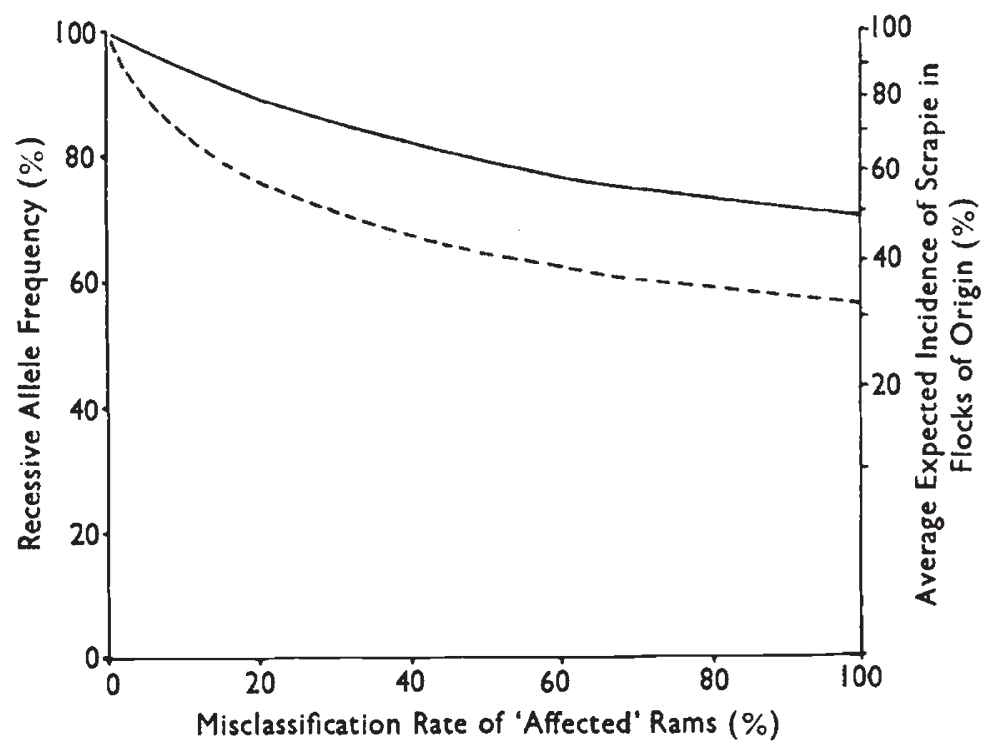

Fig. 3.-The average expected incidence of scrapie on the affected farms of origin if a recessive gene is assumed to have caused the 66 per cent. incidence in foundation stock (offspring of affected ewes). (Key: solid line, exact fit of data; broken line, lower 95 per cent. fiducial limit of data.)

It can be seen from fig. 3 that as the extent of misclassification rises the recessive allele frequency falls thereby reducing the incidence of scrapie expected on the farms of origin, in terms of this model. At the most unlikely extreme of Ioo per cent. misclassification, the average incidence is expected to be nearly 50 per cent. and even if the 95 per cent. fiducial limit of the expectation is considered, this only reduces the predicted scrapie incidence to about 30 per cent. which is still wide of the observed value of 7 per cent. Making allowance for known biases-late developing cases, commercial culling, deaths unrecognised as scrapie-it is most unlikely that the actual incidence is as much as twice the observed incidence. This model does not fit the facts.

\section{INCIDENCE IN PROGENY OF EXPERIMENTAL MATINGS}

As with the foundation stock, the overall picture is again one of too much scrapie to reconcile with a recessive gene as the sole cause. 
Every ram used for experimental matings, eleven in all, whose progeny have attained appropriate ages, has had some of them affected and five of these rams were deliberately chosen to be the

TABLE !

Scrapie incidence in progeny $*$ of affected ewes mated with two types of ram

\begin{tabular}{|c|c|c|c|}
\hline \multirow{2}{*}{ Mating } & \multicolumn{3}{|c|}{ Progeny } \\
\hline & $n$ & Unaffected & Affected \\
\hline 5 unaffected OF rams $\times$ affected ewes & $3^{\mathrm{I}}$ & I 9 per cent. & 8I per cent. \\
\hline 5 affected rams $\times$ affected ewes. & 21 & 5 & 95 \\
\hline
\end{tabular}

* All unaffected progeny $4 \frac{1}{2}$ years old or older.

"freest of the free" in terms of the criteria adopted for selecting OF stock. Despite this screening, the four OF rams with sufficient numbers of progeny for genetical testing have produced a greater proportion of affected progeny when mated with affected ewes than would be expected even if each was postulated to be a heterozygote $(\mathrm{P}<0 \cdot 05)$. The four rams have remained unaffected, three dying at $6,6 \frac{1}{2}$ and $8 \frac{1}{2}$ years of age, the other being still alive at 8 years old. Details of these results are given in table $I$ along with the results of affected $\times$ affected matings for which the incidence is very high indeed.

TABLE 2

$2 \times 2$ experiment: scrapie incidence in $O F$ and $O S$ parents and progeny * of four mating types

\begin{tabular}{|c|c|c|c|}
\hline 7 & \multicolumn{2}{|l|}{ OF } & OS \\
\hline OF & $\begin{array}{l}\mathrm{FF} \\
\text { 10 } \text { o parents: } \\
3 \text { o parents: } \\
27 \text { progeny: }\end{array}$ & $\begin{array}{c}\text { er cent. } \\
\text { ", }\end{array}$ & $\begin{array}{l}\text { SF } \\
\text { I9 } q \text { parents: } 75 \text { per cent. } \\
3 \text { o parents: o ", } \\
3^{2} \text { progeny: } 69,\end{array}$ \\
\hline os & $\begin{array}{l}\text { FS } \\
\text { I I } \& \text { parents: } \\
5 \text { o parents: } 4 \\
3^{8} \text { progeny: I }\end{array}$ & $\begin{array}{l}\text { er cent. } \\
, "\end{array}$ & $\begin{array}{l}\text { SS } \\
24 \& \text { parents: } 4^{8} \text { per cent. } \\
5 \text { O parents: } 4^{0} \quad, \\
4^{6} \text { progeny: } 4^{1}\end{array}$ \\
\hline
\end{tabular}

- All unaffected animals $4 \frac{1}{2}$ years old or older.

Many of the matings used in table I formed part of a larger series comprising all four types of mating between OS and OF groups, including reciprocal crosses. The results for this larger series are summarised in table 2. 
Several points concerning the results in table 2 require explanation. The progeny groups contain approximately equal numbers of each sex but almost all the males were castrated. Every female progeny was given the opportunity to have two crops of lambs by an unrelated OF ram. There is no indication of a sex difference in scrapie incidence. Only two of the five OS rams are known to have developed scrapie; of the others, two are still unaffected, but the third unfortunately died early without signs of scrapie though its surviving twin which was not bred from did develop scrapie (only 5 per cent. of the progeny are from this third ram and their exclusion does not affect the trend of the results). The OF rams were three of those detailed above and of the OF ewes involved, none developed scrapie.

The allocation of ewes to the various blocks was at random, each contributing successive progeny to the same block. However, in the case of OS ewes, there was a disproportion in their scrapie incidence between the two blocks: 75 per cent. of mothers of SF progeny developed scrapie, whereas only $4^{8}$ per cent. of mothers of SS lambs did so (much of this difference arose from ram infertility in the SS block matings in the first year which resulted in a number of ewes originally allocated to this block dying of scrapie before they had produced any lambs). This disproportion is suggested as the main reason for the lower incidence of scrapie in the SS animals than in the SF ones. When the incidence among dams of the SS block is adjusted to that of the SF block dams, the SS progeny incidence becomes 64 per cent. instead of $4 \mathrm{I}$ per cent., if a linear proportionality is assumed-a procedure which may only have crude validity but with some justification from the previous results.

In the light of these remarks it is concluded from table 2 that the type of ram had little effect on the variation, any small differences due to ram-type are in the opposite direction to those expected on any solely genetic hypothesis. Instead, the main source of variation appears to stem from the type of ewe and this effect is consistent with progeny from both types of ram. Incidentally, the appearance of scrapie in progeny of $\mathrm{OF} \times \mathrm{OF}$ matings disposes of the possibility that a simple dominant gene causes the disease.

Another point about this experiment affecting possible interpretations must be stated. For reasons given earlier, the OF and OS ewes were loosely isolated from one another and the same applied to their progeny, FF and FS blocks being separate from SF and SS blocks at first. Since the total experiment was built up from 4 successive lamb crops, this initial separation lasted for $3 \frac{1}{2}$ years for the earliest lamb crop and was progressively shorter for successive crops.

\section{SCRAPIE INCIDENCE IN STOCK REARED IN VARIOUS DEGREES OF CONTACT WITH THE DISEASE}

It has been impossible to determine what effect, if any, the above separation of OF and OS ewe blocks had on the results. In an attempt 
to cover this point in the early stages of the work, a numerically small experiment was set up to check the possibility of contact transmission. The small numbers-four ewes chosen at random from the OF group -were obligatory because of the extreme shortage of OF stock. At $2 \frac{1}{2}$ years old they were placed in continuous lifetime contact at pasture with as many cases of natural Suffolk scrapie as were available and the affected animals were continually replaced with fresh ones as the advanced cases had to be killed.

The introduced OF ewes were mated each year either to OF rams or unaffected FF rams: scrapie has now occurred in 40 per cent. or more of the immediate progeny of each of the four ewes, one of which has also developed the disease at 5 years old. The numbers (table 3, group a) are too small to draw firm conclusions since the genetical equivalence of the "maximum contact" and "partially isolated" groups is uncertain. However, the average incidence here of 57 per cent. scrapie compared with 18 per cent. for broadly similar contemporary stock in partial isolation (table 3 , group $d$ ) is sufficient cause to take the possibility of contact transmission seriously, the difference being significant $(\mathrm{P}<0 \cdot 02)$.

Two additional unaffected $O F$ rams not used for the original $2 \times 2$ experiment were used to provide supplementary FF type progeny to be reared along with ones in the $2 \times 2$ experiment, that is, in known contact with the disease. The degree of contact may have been different from the previous " maximum contact" group since these supplementary animals were only grazing along with early clinical cases which were continually being removed to be placed with the maximum contact group. One of the additional OF rams had previously mated with many types of ewes, including affected ones, and his FF progeny-eight in all-were placed in contact with early cases after weaning. Three of these eight have developed scrapie and the youngest survivors are now 5 years old (table 3 , group $b$ ). Twenty supplementary FF progeny from the second additional ram were born, and have remained, in contact with the $2 \times 2$ experiment stock and five of these have developed the disease by four years old (table 3, group c). None of the OF parents has so far become affected.

Also, three independent groups of isolated FF type stock were set up. These were produced from a series of OF and FF rams and ewes, almost all of which had no relationship with ones in the $2 \times 2$ experiment, except for common flocks of origin. The isolation amounted to the use of wide geographical separation from the other scrapie experiments, isolation from commercial sheep, immediate removal for close observation of any individual which showed the slightest clinical signs resembling scrapie, and provisional removal of progeny of any suspicious ewes. Scrapie has occurred in two sheep from one isolate, one of which being a ram lamb was segregated from the flock when it was weaned, and in one sheep at a second isolate. Thus three cases have occurred in a total of twenty-three 
animals born four or more years ago, while no cases have so far developed in the nineteen three-year-olds at the three locations (table 3 , group $e$ ). The three affected progeny were all born shortly before the geographical separation from other scrapie experiments was implemented in 1960. Again, none of the OF animals has developed scrapie.

No final conclusions are possible which would presuppose the genotypic parity of the various isolation and contact groups, since they are only broadly similar due to the four scrapie-free flocks of origin held in common. Nor, at this juncture, is there any real understanding of what conditions might be necessary for degrees of effective isolation or contact. But, having stated this, it is noteworthy

TABLE 3

The incidence of scrapie in FF stock reared in various degrees of contact with clinically affected stock or in attempted isolation

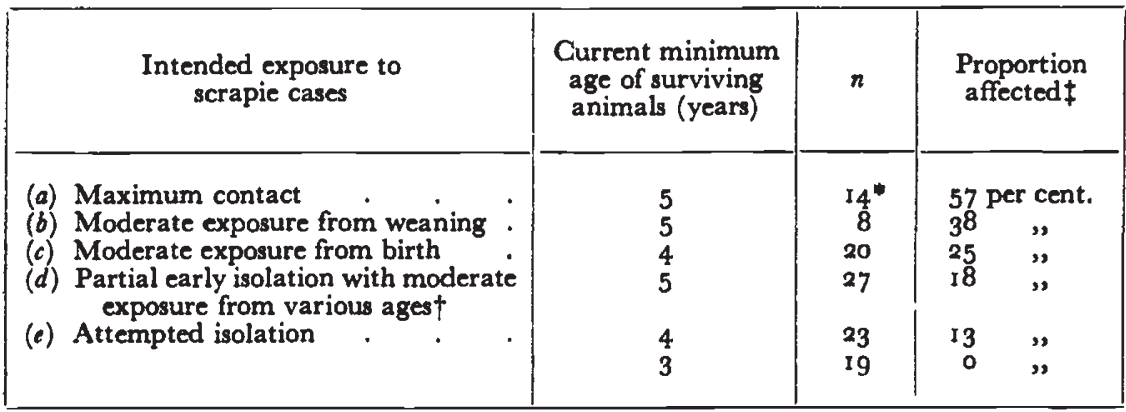

* No neuropathological examination possible for one typical clinical case (accepted as positive): of the animals considered unaffected one died at $2 \frac{1}{8}$ years and another at 3 years old.

+ From table 2.

† Apart from the exceptions noted, all unaffected animals were at least $4 \frac{1}{\frac{1}{2}}$ years old.

that the incidence in the various groups is directly proportional to the only forms of contact with the disease which can be manipulated experimentally at present, namely, exposure to clinical cases and to the progeny of affected ewes.

To the extent that these and the previous data can be interpreted as evidence of contagion, they point to the greater risk arising from contact occurring early in life.

\section{GENERAL DISCUSSION}

The need to postulate the involvement of a pathogen has been brought out in connection with the analysis of the separate aspects of the data and needs little further elaboration. Other types of work have also shown that a pathogenic organism is likely to be involved and the detailed reasons are cited in the introduction. Briefly these concern the oral transmission and contagious spread of the disease in several species, and the appearance of scrapie in free sheep placed 
on previously affected farms in Iceland (Palsson and Sigurdsson, 1958).

There is little doubt as a result of this investigation that scrapie is maternally transmitted and that all the sires used irrespective of any previous connection with the disease left a proportion of affected offspring.

Two points arise from the present work which must be discussed, one being the question of what genetic control of susceptibility to the pathogen can be inferred from these experiments, the other being the need to explain the results of some matings, notably affected $\times$ affected if a pathogen causes the disease. The latter point will be dealt with first.

There seems little doubt that when two affected Suffolk sheep are mated their progeny almost always develop scrapie within four years. The same applies where the manifestation of the disease is delayed in either or both parents for a year or two after the progeny are born, but with a disease of prolonged incubation, like scrapie, there is hardly any point in distinguishing this class from the former until more is known of the presumed infectious period.

If a particular infectious disease always appears in progeny of affected $\times$ affected matings then the organism which causes it must either be highly infectious and ubiquitous thus ensuring that all susceptible animals develop the disease or some mechanism must be present for the non-genic transmission of the infecting agent from the parents to the progeny and the prolonged connection between mother and offspring would offer the most obvious route. The necessity to infer maternal transmission in the case of scrapie in many Suffolks comes not from the affected $\times$ affected mating class, though they presumably have it, but rather from a comparison of reciprocal cross matings where only one parent has the disease. The evidence given points to the scrapie incidence in the progeny matching the mother's category. Furthermore some published information on natural scrapie can be interpreted the same way. Thus Parry's evidence on scrapie in twins shows two effects ( 1962 , table 5); first, affected ewes $\times$ unaffected rams produce 86 per cent. scrapie progeny ( $n=14$ ) whereas the reciprocal cross in which the male alone is affected has only 26 per cent. affected progeny $(n=27)$. Moreover, and contrary to Parry's conclusions, these same data when compared with a recessive-gene-only model have an excess of twin pairs both like their mother's category and a deficiency of pairs where only one member is affected $(\mathrm{P}<\mathrm{O} \cdot 00 \mathrm{I})$. This trend is not quite as marked in our own very limited group of twin pairs but the combined series show marked deviation towards similarity of mother and progeny and disagreement with the facts fitting a solely genetic hypothesis.

The postulate of non-genic maternal transmission of the pathogen seems the simplest explanation for the above twin effects, of the high incidences in OS (dam affected) progeny, the SF and SS progeny 
and in progeny of affected $\times$ affected matings. However, it would be unwarranted to assume that the existence of maternal transmission in many Suffolks implies that this route will operate whatever the genetic constitution of the sheep-it may be absent in some other breeds or some lines of Suffolks and it is possible that one ram found by Draper and Parry (1962) confers on its offspring either a genetic resistance to infection from the mother or the genotype prolongs the incubation period considerably, since 18 progeny by affected dams remained free from scrapie for 3 years. However, it has been shown using several breed combinations that maternal transmission of scrapie can take place experimentally, where ewes are challenged with scrapie agent at mating time (Gordon, I960; Dickinson et al., 1964). It is, therefore, reasonable to expect, at least in some sheep, that this maternal route will be open for the natural transmission of the scrapie pathogen.

In view of the failure to find any support in the present data for a solely genetic origin of scrapie and also because of our conclusion that maternal transmission is common in the Suffolk breed, it is necessary to examine in some detail the published evidence from which Parry ( 1960,1962 ) has concluded that a recessive gene is the probable cause. There are two major sources of bias in the main paper by Parry (1962). There is a fallacy concerning the age distribution of scrapie cases. Parry deduces from the observed age incidence data that 90 per cent. of potentially affected animals have developed scrapie by $4 \frac{1}{2}$ years old, in other words at this age 10 per cent. of potentially affected animals will be misclassified as unaffected and he corrects some of his data (for the progeny but not for the parents) on this basis. However, the data are from a population subject to commercial culling and when allowance is made for this fact (Dickinson et al., $1964 c$ ) the error rate at $4 \frac{1}{2}$ years is one in four instead of one in ten and this corrected figure is presumably an underestimate if, as we conclude, scrapie can be contagious. With such a large proportion of misclassification, especially as it affects the parental categories, it is doubtful how much reliance can be placed on any scheme which depends on classifying the individuals into different mating types.

Secondly, there is also a potentially circular argument arising from allocating presumed genotypes to rams on the basis of the presence or absence of scrapie in the progeny. The presence of a recessive gene is then deduced from the incidence of scrapie in the different types of mating fitting Mendelian theory: but the basis on which most of the matings are classified as being different in the first place is related to the incidence of scrapie in the progeny. Obviously bias from this circular argument is virtually absent if family size is very large but at small family size it is extreme. No clear indication of the ram family sizes is given and it is difficult therefore to estimate the seriousness of the bias but some indication that it does affect the 
issue comes from the absence of some of the possible types of matings: the 442 progeny (Parry, 1962; table 2) are confined to 7 of the 9 possible types of mating. The missing classes both involve unaffected rams (supposed dominant homozygotes) in the one case mated with unaffected females (supposed heterozygotes) and in the other with affected females. If maternal transmission occurs widely this could account for the absence of the latter class, due to the methods of classification which Parry uses. The same explanation coupled with bias due to late manifestation of scrapie could lead to a deficiency in the former class. But, on the basis of our evidence, rams which do not have affected progeny are rare and this, whatever its interpretation, would lead to the absence of these two classes. On the same grounds the mating class involving only supposed dominant homozygotes should be deficient and it is significant that only 4 per cent. of the matings are shown in this class.

Parry accounts for this rarity of matings involving supposed homozygous dominant rams by proposing that the breeders select against such rams but there is no evidence for this. If the figures given are a fair sample of the flocks used then the frequency of the postulated dominant allele in the ewes appears to be in the order of 7o-8o per cent. which is hard to reconcile with this proposed selection. On the contrary the only meaningful evidence on the point is that there is natural selection against the genotypes of affected animals. The known selection is severe in the case of affected females, since they commonly die young and obviously leave few progeny compared with unaffected animals, and this presumably could account for the preponderance of matings with unaffected females in Parry's data. Natural selection on the males is likely to have a relatively smaller effect than with the females on changes in any relevant allele frequencies, since in many flocks males are used young and often disposed of by 4 years old in any case. If the breeders select against supposed homozygous dominant rams, it is relevant to point out that most of the eleven rams in our experiments were breeders' rejects on conformation grounds and yet all have left affected progeny.

Returning to the question raised earlier concerning genetical control of susceptibility: for natural scrapie the evidence on this is inconclusive. This is not surprising if, as appears, the occurrence of exposure to the agent is haphazard and uncertain and it may well be important to take into account the age at the time of exposure. This factor of exposure age could be very important in assessing the apparently dominant role of maternal transmission and any genetical control of susceptibility at one age may not act at other ages.

At two points in the data presented there is some evidence that sires may differ in the proportion of their progeny which develop scrapie: the OS (sire affected) group were unanalysable because of this sire heterogeneity and the incidence of scrapie in progeny of affected mothers is lower, though not significantly so, where the sire 
is unaffected than where he is affected. Whether or not these features are evidence of genetical differences among the sires remains uncertain but, whatever the explanation, the variation between sires is a small component in comparison with the large effect due to maternal transmission.

It is an open question whether any inferences regarding susceptibility to natural scrapie can be drawn from work on induced scrapie since there is some evidence for breed and agent differences in this respect (Dickinson, unpublished). However, there is evidence for a considerable degree of genetic control of susceptibility to certain specific forms of induced scrapie (Dickinson et al., 1965; Gordon, 1965) though this is probably not simply controlled by a major gene.

A high degree of genetical control of incubation period of scrapie in mice has been demonstrated (Dickinson and Mackay, 1964). The incubation periods span a twofold range (20-40 weeks) according to the genotype of the mouse and a similar diversity has been found in Cheviot sheep injected with scrapie, where a proportion of susceptible animals take over two years to manifest the disease (Dickinson, unpublished). It is a matter for speculation what bearing, if any, this particular phenomenon has on the results analysed here for natural scrapie but in order to cover this type of problem we have been careful to retain all unaffected animals until they were senile.

This recent work is therefore bringing to light further complexities in the genetical aspects of the disease and it serves to emphasise some of the practical difficulties implicit in further studies of natural scrapie.

\section{SUMMARY}

Analysis of the incidence of natural scrapie both in experimentally bred Suffolk sheep and in samples from private flocks, leads to the firm conclusion that the disease is not caused by the action of a simple recessive or dominant gene in the sheep.

The total evidence on scrapie is shown to be consistent with the interpretation that a biologically independent pathogenic organism causes the disease and that in some types of sheep at least there is vertical transmission of this organism from mother to offspring which contributes to correlated incidences of the disease in some classes of relatives and hence the superficial appearance of a genetically determined origin of the disease.

The evidence, both direct and indirect, for contagious spread and maternal transmission of scrapie is discussed. The difficulties in using field data for this type of work are specified and the scope of potential genetical variation likely to affect the disease is reviewed.

Acknowledgments.-This work could not have been undertaken without the willingness of Dr I. Zlotnik to carry out a neuropathological examination of all the animals, and it is a pleasure to record our indebtedness to him and his staff. We wish to record our appreciation of the important part played by $\mathrm{Mr}$ W. Buist in the initiation and management of the breeding flocks and of the management 
subsequently carried out by $\mathrm{Mr}$ J. Brown. We would also like to thank $\mathrm{Dr} \mathrm{St}$ C. S. Taylor for many helpful discussions during the analysis of the data. This work was carried out in part with a grant from the United States Department of Agriculture administered under PL 480.

\section{REFERENCES}

CHANDLER, R. L. 1961. Encephalopathy in mice produced by inoculation with scrapie brain material. Lancet, $t, 1378-1379$.

CHANDLER, R. L., AND FISHER, J. 1963. Experimental transmission of scrapie to rats. Lancet, $2, I_{1} 65$.

CHELle, P. L. 1942. Un cas de tremblante chez la chevre. Bull. Acad. vét. Fr., I5, 294-295.

DICKINSON, A. G., AND MACKAY, J. M. K. 1964. Genetical control of the incubation period in mice of the neurological disease, scrapie. Heredity, 19, 279-288.

DiCKinson, A. G., MACKAY, J. M. K., AND ZzotNiK, 1. 1964. Transmission by contact of scrapie in mice. J. comp. Path., 74, 250-254.

Dickinson, A. G., YounG, G. B., STAMP, J. T., AND RENWick, c. c. 1964. A note on the distribution of scrapie in sheep of different ages. Anim. Prod., 6, 375-377.

Dickinson, A. G., YounG, G. B., AND RENWick, c. C. 1965. Scrapie: experiments involving maternal transmission in sheep. Scrapie Seminar Proceedings, U.S.D.A., A.R.S., in press.

DRAPER, G. J., AND PARRY, H. B. 1962. Scrapie in sheep; the hereditary component in a high incidence environment. Nature, 195, 670-672.

Gordon, w. S. 1960. Report of Special Meetings on Scrapie, U.S.D.A., A.R.S., 91-22, I-I 2.

GORDoN, w. s. 1965. Variation in susceptibility of sheep to scrapie and genetic implications. Scrapie Seminar Proceedings, U.S.D.A., A.R.S., in press.

GORDON, W. S., AND PATTISON, I. H. 1957. The experimental production of scrapie in goats. Vet. Rec., 69, 1444 .

MACKAY, J. M. K., SMith, W., AND STAMP, J. T. 196o. Experimental scrapie: some recent work. Vet. Rec., 72, 1002-1006.

MACKAY, J. M. K., AND SMITH, W. I961. A case of scrapie in an uninoculated goat -a natural occurrence or a contact infection? Vet. Rec., 73, 394-396.

MCDANIEL, H. A., AND MOREHouse, L. G. 1963. The diagnosis of scrapie. 67th Ann. Proc. U.S. Livestock Sanitary Assoc., pp. 550-564.

PALSSON, P. A., AND SIGURDSSON, B. 1958. Rida: a slow progressive disease affecting the central nervous system of sheep. Proc. 8th Nord. Vet. cong., A8, 1 79-19I.

PARRY, H. B. 1960. A transmissible hereditary disease of sheep. Nature, 185, $44^{1-443 .}$

PARRY, H. B. 1962. Scrapie: A transmissible and hereditary disease of sheep. Heredity, $17,75-105$.

PATtison, I. H., AND millson, G. C. I961a. Scrapie produced experimentally in goats with special reference to the clinical syndrome. F. comp. Path., $7 t$, IOI-108.

Pattison, I. H., AND millson, G. C. 196I $b$. Experimental transmission of scrapie to goats and sheep by the oral route. J. comp. Path., 7x, 1 71-176.

Pattison, I. H. 1964. The spread of scrapie by contact between affected and healthy goats, sheep and mice. Vet. Rec., 76, 333 .

STAMP, J. T., BROTHERSTON, J. G., ZLOTNIK, I., MACKAY, J. M. K., AND SMITH, W. 1959. Further studies on scrapie. J. comp. Path., 69, 268-280.

STAmp, J. T. 1962. Scrapie: a transmissible disease of sheep. Vet. Rec., 74, 357362.

stamp, J. T. 1965. Scrapie: A review of the problem. Scrapie Seminar Proceedings, U.S.D.A., A.R.S., in press.

WILSON, D. R., ANDERSON, R. D., AND SMITH, w. 1950. Studies in scrapie. F. comp. Path., 6o, 267-282. 
zLotNIK, I. 1958. The histopathology of the brainstem of sheep affected with natural scrapie. 7. comp. Path., 68, 148-166.

zLotnik, 1. I962. The pathology of scrapie: A comparative study of the lesions in the brain of sheep and goats. Acta. neuropath. (Suppl. I), 61-70.

zLotNIK, I. 1963. Experimental transmission of scrapie to golden hamsters. Lancet, ii, I072.

ZLOTNIK, I., AND KATIYAR, R. D. Ig61. The occurrence of scrapie disease in sheep of the remote Himalayan foothills. Vet. Rec., 73, 543-544.

ZLOTNIK, I., AND RENNIE, J. C. 1962. The pathology of the brain of mice inoculated with tissues from scrapie sheep. J. comp. Path., 72, 360-365.

zLOTNIK, I., AND RENNIE, J. C: I 963 . Further observations on the experimental transmission of scrapie from sheep and goats to laboratory mice. F. comp. Path., 73, 150-162. 